Claremont Colleges

Scholarship@ Claremont

All HMC Faculty Publications and Research

HMC Faculty Scholarship

5-1-1975

\title{
A Simple Characterization of Commutative Rings Without Maximal Ideals
}

Melvin Henriksen

Harvey Mudd College

\section{Recommended Citation}

Henriksen, Melvin. "A simple characterization of commutative rings without maximal ideals." American Mathematical Monthly 82.5 (1975): 502-505.

This Article is brought to you for free and open access by the HMC Faculty Scholarship at Scholarship @ Claremont. It has been accepted for inclusion in All HMC Faculty Publications and Research by an authorized administrator of Scholarship @ Claremont. For more information, please contact scholarship@cuc.claremont.edu. 


\title{
CLASSROOM NOTES
}

\author{
Edited By Richari) A. Brualdi
}

Material for this Department should be sent to R. A. Brualdi, Department of Mathematics, University of Wisconsin, Madison, WI 53706.

\section{A SIMPLE CHARACTERIZATION OF COMMUTATIVE RINGS WITHOUT MAXIMAL IDEALS}

\section{MELvin HenRIKSEN}

In a course in abstract algebra in which the instructor presents a proof that each ideal in a ring with identity is contained in a maximal ideal, it is customary to give an example of a ring without maximal ideals. The usual example is a zero-ring whose additive group has no maximal subgroups (e,g., the additive group of (dyadic) rational numbers; actually any divisible group will do; see $[1$, p. 67]). This may leave the impression that all such rings are artificial or at least that they abound with divisors of 0 .

Below, I give a simple characterization of commutative rings without maximal ideals and a class of examples of such rings, including some without proper divisors of 0 . To back up the contention that this can be presented in such a course in abstract algebra, I outline proofs of some known theorems including a few properties of radical rings in the sense of Jacobson.

The Hausdorff maximal principle states that every partially ordered set contains a maximal chain (i.e., a maximal linearly ordered subset). It is equivalent to the axiom of choice [4, Chapter $\mathrm{XI}]$.

Since the union of a maximal chain of proper ideals in a ring with identity is a maximal ideal, and since the union of a maximal chain of linearly independent subsets of a vector space is a maximal linearly independent set, we have:

(1) Every ideal in a ring with identity is contained in a maximal ideal.

(2) Every non-zero vector space has a basis.

As usual we denote the ring of integers by $Z$, and for any prime $p \in Z$, we denote by $Z_{p}$ the ring of integers modulo $p$, and by $Z_{p}^{\prime}$ the zero-ring whose additive group is the same as that of $Z_{p}$.

It is not difficult to prove that a commutative ring $R$ has no nonzero proper ideals if and only if either $R$ is a field or $R$ is isomorphic to $Z_{p}^{\prime}$ for some prime $p$. See $[5$, p. 133]. Hence:

(3) An ideal $M$ of a commutative ring $R$ is maximal if and only if $R / M$ is either a field or is isomorphic to $Z_{p}^{\prime}$ for some prime $p$.

For any commutative ring $R$, let $J(R)$ denote the intersection of all the ideals $M$

AMOR mant MONTHLY VL $82(5) 1975$ 
of $R$, such that $R / M$ is a field. If $R$ has no such ideals, let $J(R)=R$. In the latter case we call $R$ a radical ring. The knowledgeable reader will recognize $J(R)$ as the Jacobson radical of $R$. See [2, Chapter 1].

Of the many known properties of radical rings, we need only the following two, the first of which follows immediately.

(4) A homomorphic image of a (commutative) radical ring is a radical ring.

(5) $J(R)$ is a radical ring.

Proof. If $J(R)$ is not a radical ring, then there is a homomorphism $\phi$ of $J(R)$ onto a field $F$ with identity element 1 . Choose $e \in J(R)$ such that $\phi(e)=1$, and define $\phi^{\prime}: R \rightarrow F$ by letting $\phi^{\prime}(a)=\phi(a e)$ for each $a \in R$. If $a, b \subseteq R$, then

$$
\begin{aligned}
\phi^{\prime}(a+b) & =\phi((a+b) e)=\phi(a e+b e)=\phi(a e)+\phi(b e)=\phi^{\prime}(a)+\phi^{\prime}(b), \\
\text { and } \phi^{\prime}(a b) & =\phi(a b e)=\phi(a b e) \phi(e)=\phi(a e b e)=\phi(a e) \phi(b e)=\phi^{\prime}(a) \phi^{\prime}(b) .
\end{aligned}
$$

Therefore $\phi^{\prime}$ is a homomorphism of $R$ onto $F$, and hence its kernel contains $J(R)$. But $e \in J(R)$ and $\phi^{\prime}(e)=1$. This contradiction shows that $J(R)$ is a radical ring.

It follows easily from (1), (3), and (4) that no ring with identity is a radical ring and that every zero-ring is a radical ring.

THEOREM. A commutative ring $R$ has no maximal ideals if and only if

(a) $R$ is a radical ring.

(b) $R^{2}+p R=R$ for every prime $p \in Z$.

Proof. Suppose first that (a) and (b) hold. Since $R$ is a radical ring, no homomorphic image of $R$ can be a field, so, by (3) it suffices to show that for any prime $p \in Z$, the zero-ring $Z_{p}^{\prime}$ is not a homomorphic image of $R$. Suppose, on the contrary, that there is a homomorphism $\phi$ of $R$ onto $Z_{p}^{\prime}$ with kernel $M$. If

$$
c=\sum_{i=1}^{n} a_{i} b_{i} \in R^{2}, \text { then } \phi(c)=\sum_{i=1}^{n} \phi\left(a_{i}\right) \phi\left(b_{i}\right)=0,
$$

so $R^{2} \subset M$. Moreover, $\phi(p a)=p \phi(a)=0$, so $p R \subset M$. Hence $R^{2}+p R \subset M \neq R$, so (b) fails. The contradiction shows that $R$ has no maximal ideals.

Suppose next that $R$ has no maximal ideals. By (3) and the definition of $J(R)$, $R$ is a radical ring. Suppose (b) fails for some prime $p$, let $I=R^{2}+p R$, and let $\phi$ be the natural homomorphism of $R$ onto $R / I$. If $a, b \in R$, then $0=\phi(a b)=\phi(a) \phi(b)$, so $R / I$ is a zero-ring, and since $0=\phi(p a)=p \phi(a)=0, R / I$ has characteristic $p$ and hence is a vector space over $Z_{p}$. By (2), since $I \neq R, R / I$ has a basis $\left\{x_{\alpha}\right\}_{\alpha \in \Gamma}$ and each $x \in R / I$ may be written uniquely as $x=\Sigma_{\alpha \in \Gamma} a_{\alpha} x_{\alpha}$ with $a_{\alpha} \in Z_{p}$ and $a_{\alpha}=0$ for all but finitely many $\alpha \in \Gamma$. For any fixed $\alpha_{0} \in \Gamma$, the mapping $\psi_{0}$ such that $x \psi_{0}=a_{\alpha_{0}}$ is a homomorphism of $R / I$ onto $Z_{p}^{\prime}$. Then $\phi \circ \psi_{0}$ is a homomorphism of $R$ onto $Z_{p}^{\prime}$. By (3), the kernel of $\phi_{\circ} \psi_{0}$ is a maximal ideal, contrary to assumption. Hence (a) and (b) hold. 
Recall that an abelian group $G$ is divisible if $n G=G$ for every $n \in Z$ and note that $G$ is divisible if and only if $p G=G$ for every prime $p \in Z$. It follows from the theorem that a zero-ring whose additive group is divisible has no maximal ideals.

COROLLARY. Let $S$ be a commutative ring with identity that has a unique maximal ideal $R$. If $R^{2}+p R=R$ for every prime $p \in Z$, then $R$ has no maximal ideals. In particular, if the additive group of $S$ is divisible, then $R$ has no maximal ideals.

I conclude with some explicit examples:

Examples. (i) For a field $F$, let $F[x]$ denote the ring of polynomials in an indeterminate $x$ with coefficients in $F$, and let $F(x)$ denote the field of quotients of $F[x]$. Let

$$
S(F)=\left\{h(x)=\frac{f(x)}{g(x)} \in F(x): f(x), g(x) \in F[x] \text { and } g(0) \neq 0\right\} .
$$

It is easy to verify that $S(F)$ is an integral domain whose unique maximal ideal is $R(F)=x S(F)$. If $F$ has characteristic zero, then, by the corollary, $R(F)$ has no maximal ideals. If $F$ has prime characteristic, then, since $[R(F)]^{2}=x^{2} R(F)$, the ring $R(F)$ does have maximal ideals.

(ii) Let $G$ denote the additive semigroup of non-negative dyadic rational numbers, and let $U(F)$ denote the semigroup algebra over $G$ with coefficients in a field $F$. We may regard each element of $U(F)$ as a polynomial in $x^{\left(\frac{1}{2}\right)^{n}}$ for some positive integer $n$. Let $T(F)$ denote those elements of the quotient field of $U(F)$ whose denominators fail to vanish at 0 . It is not difficult to verify that $R^{*}(F)$ $=\{h \in T(F): h(0)=0\}$ is the unique maximal ideal of $T(F)$ and that $\left[R^{*}(F)\right]^{2}$ $=R^{*}(F)$. By the corollary, $R^{*}(F)$ has no maximal ideals (and no proper divisors of 0 ).

(iii) Let $F_{1}$ be a field of characteristic 0 , let $F_{2}$ be a field of prime characteristic $p$, and let $R$ be the direct sum of the ring $R\left(F_{1}\right)$ described in (i) and the ring $R^{*}\left(F_{2}\right)$ described in (ii). Since each of these latter two rings is a radical ring, so is $R$. For, otherwise, there would be a homomorphism $\phi$ of $R$ onto a field $F$. Then $\phi\left[R\left(F_{1}\right)\right]$ and $\phi\left[R^{*}\left(F_{2}\right)\right]$ are ideals of $F$ whose (direct) sum is $F$, and hence one of them is all of $F$, contrary to the fact that $R\left(F_{1}\right)$ and $R^{*}\left(F_{2}\right)$ are radical rings. Also, while $R^{2} \neq R$ and $p R \neq R$, it is true that $R^{2}+p R=R$, so $R$ has no maximal ideals.

One can create more rings satisfying the hypothesis of the corollary by starting with any commutative ring $S$ with identity and divisible additive group, taking its localization $S_{M}$ at a maximal ideal $M$, and letting $R=M S_{M}$. See [1, Chapter 2].

I am grateful to Professor Barbara Beechler for valuable criticisms of earlier drafts of this note.

\section{References}

1. I. Fuchs, Abelian Groups, Hungarian Academy of Sciences, Budapest, 1958. 
2. I. N. Herstein, Noncommutative rings, MAA - The Carus Mathematical Monograph No. $15,1971$.

3. S. Lang, Algebra, Addison-Wesley, Reading, Mass., 1965.

4. S. Warner, Modern Algebra, Vol. II, Prentice-Hall, Englewood Cliffs, N. J., 1965.

5. O. Zariski and P. Samuel, Commutative Algebra, Vol. I, Van Nostrand, Princeton, N. J., 1958.

Department of Mathematics, Harvey Mudd College, Claremont, Ca 91711. 